\title{
DIMENSIONANDO LA PRECARIEDAD LABORAL EN MÉXICO DE 2005 A 2015, A TRAVÉS DEL MODELO LOGÍSTICO ORDINAL GENERALIZADO
}

Measuring precariousness at work in Mexico from 2005 to 2015, through Generalized Ordinal Logistic Model

Jorge Alberto Pérez Cruz ${ }^{1}$, Guadalupe Isabel Ceballos Álvarez²

Fecha de recepción: 31 enero de 2017

Fecha de aceptación: 04 junio de 2017

1- Nombre: Jorge Alberto Pérez Cruz. Nacionalidad: Mexicana. Grado: Doctor. Especialización: Ciencias Económicas. Adscripción: Universidad Autónoma de Tamaulipas. Correo electrónico: doc.jorgeperez@gmail.com 


\section{Resumen}

En México existe una mayor proporción de la población con mejores niveles educativos que hace una década; sin embargo, esto no ha contribuido a elevar los niveles de vida de los trabajadores. La evidencia empírica encontrada a través del Modelo Ordinal Generalizado muestra que en 2015 en comparación con 2005 se ha incrementado la probabilidad de que las condiciones laborales en México se precaricen, esto es que existe una mayor proporción de trabajadores que experimentan una disminución en el salario, una disminución en las prestaciones laborales y un aumento en las jornadas de trabajo, sin importar el sector productivo donde labore, el tamaño de empresa, la edad, sexo, estado civil y el grado educativo.

Palabras Clave: Precariedad laboral, salarios, jornada de trabajo, prestaciones laborales y educación.

\section{Abstract}

In Mexico, there is a greater proportion of the population with better educational levels than a decade ago; however, this has not contributed to raise the living standards of workers. The empirical evidence found through the Generalized Ordinal Model shows that in 2015, compared to 2005, the probability of precarious conditions in Mexico has increased, that is, there is a greater proportion of workers experiencing a decrease in wages, a decrease in work benefits and an increase in working hours, regardless of the productive sector where they work, company size, age, sex, marital status and educational level.

Keywords: Precarious work, wages, working hours, labor benefits and education. 


\section{Introducción}

E

n la actualidad, se percibe un panorama económico mundial poco alentador que ha sido producto de la inestabilidad económica en los últimos 15 años, caracterizado por constantes recesiones económicas y recientemente por la crisis financiera de 2009 que tuvo efectos internacionales. Esto ha tenido impactos en las actividades económicas que se reflejan principalmente en su productividad y como consecuencia en la competitividad de las empresas en los mercados. Lo anterior ha expuesto la necesidad de fortalecer a las empresas para enfrentar contextos tan adversos como los observados en los últimos años. Una estrategia implementada por las empresas para hacer frente a estos contextos, no tan solo en México, sino que también en el mundo, ha sido a través de promover políticas que flexibilizan al mercado de trabajo, lo que ha representado un mecanismo para mejorar la competitividad de estas y con ello impulsar el desarrollo económico del país.

En este sentido, la flexibilización del mercado de trabajo ha sido concebida como una condición necesaria para elevar los niveles de empleo y mejorar el desarrollo económico; consolidándose como una "nueva válvula de escape"; sin embargo, con la flexibilización se crean empleos, pero pone en duda la calidad de los mismos. De hecho, no existen resultados que expongan los beneficios que ha dejado para la economía la flexibilización del mercado de trabajo (Zúñiga, 2012), estos no se observan en países desarrollados y mucho menos en países como México.

Por el contrario, la flexibilidad del mercado de trabajo -en conjunto con el complejo entorno económico- ha provocado que las condiciones de trabajo de la población se hayan deteriorado, generando con ello empleo precario (Mora-Salas y Oliveira, 2009). Las condiciones de trabajo se han deteriorado debido a que las jornadas de trabajo son más prolongadas, se han perdido prestaciones laborales, los sindicatos han perdido fuerza de negociación, ausencia de contratos, se ha incrementado la contratación eventual, entre otras (Anguiano y Ortiz, 2013; Román, 2013). Aunque en el tema de los sindicatos, aun cuando se han debilitado los mecanismos de negociación, los trabajadores que se encuentran afiliados a estos, reciben mejores condiciones laborales que los que no están afiliados (Rubio, 2017). Estas características describen la precariedad del empleo y se alejan de la concepción de un empleo asalariado típico, caracterizado por jornadas de tiempo completas, laborar en un solo lugar, asignado a un empleador, apoyo sindical y protegido por la ley laboral (Fernández, 2014).

El mecanismo de subcontratación no es nuevo, ha existido en periodos pasados; no obstante, con la flexibilidad del mercado laboral, se ha extendido a las diversas áreas de las empresas; en el pasado se acotaba a labores de seguridad, limpieza y comedor para los trabajadores (Barattini, 2009). Este mecanismo ha permitido a las empresas mejorar sus condiciones de competitividad, ya que reducen costos de producción. Sin embargo, con este tipo de contratos, el trabajador ha perdido la estabilidad laboral, de esta manera queda expuesto directamente a los efectos de las recesiones y crisis económicas (Zúñiga, 2012).

No se puede considerar que los trabajadores experimentan de manera uniforme la precariedad; algunos podrían tener contrato temporal y no gozar de prestaciones; otros podrían trabajar jornadas excesivas fuera de lo que establece la ley, mientras que otros podrían percibir bajos ingresos. Por ello resulta sustancial identificar las formas en que se podría presentar. Al respecto, Román (2013) menciona tres 
dimensiones que permiten contextualizar el fenómeno de la precariedad en el empleo. La primera se refiere al aspecto económico que incluye la disponibilidad del trabajo y la pertinencia de los ingresos que percibe el trabajador para poder subsistir. La segunda dimensión contempla la normatividad que señala los derechos de los trabajadores; y la tercera, el acceso a la seguridad social. Cada una de estas dimensiones refleja las condiciones deficientes del trabajador.

Los indicadores construidos por El Consejo Nacional de Evaluación de la Política de Desarrollo Social (Coneval) y el Observatorio de Salarios de la Universidad Iberoamericana de Puebla, señalan que las condiciones salariales de México han cambiado, particularmente se ha observado una pérdida en el poder adquisitivo de la población, en donde el nivel educativo no ha representado un factor que ejerza diferenciación en cuanto a dicha pérdida, dado que al igual que la población de bajos niveles educativos, han experimentado una menor capacidad de compra. También la modalidad de contratación laboral ha transitado a mayores niveles de precariedad en México, dado que la proporción de personas que se contratan sin seguridad social ha crecido en los últimos años, lo que significa que no tienen derechos para acceder a un crédito para vivienda (con subsidios del Estado), ni a seguro médico y mucho menos la posibilidad de pensionarse.

En este sentido, es importante dimensionar la problemática de la precariedad laboral que se presenta en México, mostrando evidencia que respalde el hecho que nos encontramos en un escenario donde el valor que se le asigna al trabajo realizado ha disminuido, deteriorando las condiciones laborales de los trabajadores y afectando su bienestar. De esta manera, en este trabajo se pretende establecer una forma de clasificar y dimensionar las diferentes expresiones de precariedad a través de las condiciones de la demanda de trabajo que experimentan los trabajadores en los diversos sectores en el país, contraponiéndola con aspectos de la oferta y de la misma demanda de trabajo para describir cómo esta condición de precariedad se vincula con los niveles educativos, la mayor incorporación de la mujer al mercado de trabajo, el estado civil, la edad, el sector donde se labora así como el tamaño de la empresa. Los indicadores que se presentan para contextualizar la problemática de la precariedad del trabajo en México, se realizaron utilizando información del primer trimestre de la Encuesta Nacional de Ocupación y Empleo (ENOE) 2005 y 2015, con una cobertura nacional.

El trabajo se estructuró en cinco partes. La primera es la introducción; posteriormente se expone la forma en que se ha abordado el tema de la precariedad del trabajo; en la tercera parte se explica la forma en que se construyeron los indicadores del presente estudio, para posteriormente presentar los resultados de este. Finalmente, las conclusiones del trabajo de investigación.

\section{Perspectivas de la precariedad laboral}

La precariedad laboral puede ser analizada a partir de una visión de la oferta que implica revisar aspectos relacionados con las características de los trabajadores, tales como la edad, sexo, nivel académico, estado civil, entre otros. Pero también puede ser analizada por el lado de la demanda, la cual se asocia a las condiciones del mercado productivo, respecto al tipo de actividad económica, tamaño de la unidad económica, jornada de trabajo y condiciones laborales (Mora-Salas y Oliveira, 2009). A través de las tres dimensiones que se mencionaron en el apartado anterior, se pueden identificar que la forma de la 
precariedad laboral se enfoca más en una visión de la demanda, debido a que son las empresas con el respaldo del estado quienes, de acuerdo a sus estrategias de negocios y la incorporación de nuevos procesos tecnológicos, determinan las condiciones laborales de los trabajadores (Barattini, 2009; Román, 2013).

El trabajo productivo sigue siendo fundamental para el crecimiento económico de las diferentes actividades productivas, en este aspecto no queda duda, el problema surge debido a que cada vez las empresas, en su búsqueda de lograr ser más competitivas, implementan estrategias que les permitan ser más eficientes en costos, por lo que deciden reducir beneficios a los trabajadores, incrementar jornadas de trabajo, reducir personal y reducir salarios (Anguiano y Ortiz, 2013; Barattini, 2009; Espinosa y Hernández, 2013; Solís, 2014). Los trabajadores han quedado al desamparo, los sindicatos cada vez intervienen menos para defender los derechos laborales de los trabajadores (Rubio, 2017), principalmente porque se priorizan intereses de grupos políticos y empresariales; en consecuencia, en la actualidad, la incorporación del trabajador al mercado se vuelve más incierta y vulnerable (Espinosa y Hernández, 2013; Solís, 2014). Al respecto, Rubio (2017) expone que el personal sindicalizado en México ha disminuido; en una década, la proporción de hombres que se encuentran sindicalizados pasó de 15 por ciento en 2005 a 12 por ciento en 2014, y en el caso de las mujeres, pasó de 19.4 a 16 por ciento en el mismo periodo. Esto significa que ha afectado en mayor medida a los hombres que a las mujeres.

El problema de la precariedad laboral no se asocia directamente a un contexto de economía informal, ya que se encuentra presente también en la economía formal. Con la reforma laboral que institucionalizó el estado mexicano en 2013, se flexibiliza aún más el mercado de trabajo para mejorar la competitividad de las empresas y generar en el mercado formal mayor empleo. Con esta reforma, no se espera que los nuevos empleos sean de calidad, por el contrario, se espera que se creen empleos en condiciones más precarias. Sin embargo, como lo expone Barattini (2009), la precariedad podría ser mayor en la economía no estructurada, ya que en la informalidad predominan salarios bajos y se carece de seguridad social. De hecho, en el trabajo de Huesca y Ochoa (2016), señalan que la desigualdad salarial en México y en la Frontera Norte del país se debe principalmente a que ha aumentado la proporción de trabajadores que desarrollan actividades intensivas en mano de obra y de manera informal.

Por otro lado, Fernández (2014) también expone que el fenómeno de precariedad no se vincula directamente a ciertas actividades productivas de la economía. En la literatura sobre crecimiento económico se establece que existen sectores de baja productividad que se relacionan con actividades que son intensivas en mano de obra; $y$, por otro lado, existen sectores dinámicos los cuales se asocian con procesos intensivos en capital; entonces, las condiciones del empleo mejorarán para aquellos que se encuentren laborando en sectores intensivos en capital y los que laboren en sectores menos dinámicos tendrán menores remuneraciones. Sin embargo, Mora-Salas y Oliveira (2009) encuentran que existe precariedad laboral en los diversos sectores económicos, aunque varía de un sector a otro. En donde menos precariedad existe es en los sectores más dinámicos como el sector industrial. Román (2013) y Samaniego (2010) encuentran resultados similares. Por tanto, la evidencia empírica señala que la precariedad laboral aparece en todos los sectores económicos y no es privativo de uno solo o de algunos.

Si bien es cierto que la precariedad se observa en distintos grados de acuerdo al tipo de sector que se refiera, también sucede lo mismo cuando se hace mención sobre el tamaño de la empresa, siendo en los micronegocios donde el nivel de precariedad laboral se exacerba. Román (2013), en su estudio sobre precariedad laboral de los jóvenes, encuentra que existe mayor probabilidad de que los jóvenes que se em- 
plean en micronegocios experimenten condiciones laborales precarias. Aunque con el mecanismo de la subcontratación se ha generalizado a las diversas empresas, sin importar el tamaño de estas; Samaniego (2010), expone que las mayores reducciones de la masa salarial se han presentado en los establecimientos grandes.

En empresas grandes se ha precarizado el trabajo, debido a que se han modificado las formas de contratación. En el pasado se tenían contratos por tiempo indefinido; hoy en día se observa cada vez más que prevalecen los contratos por temporalidad. También en este tipo de empresas las jornadas laborales se han vueltos más extensas. En el caso de los micronegocios, las formas de la precariedad se intensifican, debido a que las jornadas tienden a ser más prolongadas, el salario es muy bajo, no se cuenta con seguridad social y, en la mayoría de los casos, no se formalizan las formas de contratación (Barattini, 2009; Mora-Salas y Oliveira, 2009; Román, 2013).

La mayoría de la literatura reciente sobre la precariedad del mercado de trabajo pone énfasis en que es la demanda la que determina las condiciones precarias de los trabajadores, pues es quien define la disponibilidad del empleo, el nivel de salario a pagarse, la duración de las jornadas de trabajo, las condiciones de empleabilidad y como es la seguridad social. Tradicionalmente se asociaba que el fenómeno de la precariedad se presentaba en poblaciones con bajos niveles de escolaridad, por cuestiones de género y por la edad. Sin embargo, los resultados presentados por Aparicio (2013), Mora-Salas y Oliveira (2009), Román (2013) señalan que la precariedad no es un rasgo característico de la mujer, o de aquellos que tienen niveles educativos bajos o de los jóvenes; este es un problema que se ha generalizado a todos los segmentos de la población sin importar su condición socioeconómica ni sociodemográfica. Aunque el grado de precariedad podría manifestarse en diferentes formas de acuerdo a sus condiciones.

En este sentido, el modelo económico actual prioriza la acumulación de capital por parte de las empresas para hacerla más productiva y competitiva, dando lugar a formas de contratación flexibles que lo que han logrado en los últimos años ha sido precarizar la condición laboral del trabajador (Julián, 2014). En la actualidad, la empresa ha dejado de lado su responsabilidad ética con el trabajador y ha colocado como su objetivo único obtener la mayor cantidad de beneficios económicos (Zúñiga, 2012). Es por esto, que la calidad del empleo depende en buena medida de las decisiones de los empresarios, y no son el reflejo de los desequilibrios de los mercados y de aspectos que se asocien con las características sociodemográficas de los trabajadores (Román, 2013).

\section{Las condiciones laborales en México entre 2005 y 2015}

En México, según datos de la Encuesta Nacional de Ocupación y Empleo (ENOE), ${ }^{3}$ para el primer trimestre de 2005 la proporción de la población mayor o igual a 15 años de edad que se encontraban laborando fue de 39.5 por ciento; esto es, 42,137,714 ocupados y en 2015 esa proporción se ubicó en 41.7 por ciento que correspondieron a 50,220,358 ocupados, lo que significó un aumento de 2.2 puntos porcentuales; es decir, esta diferencia es la cantidad de población de 15 años y más que se incorporó al mercado de trabajo en una década. De esta población, se encuentra una mayor participación de la mujer

3- Publicado por el Instituto Nacional de Estadística y Geografía (inegi) 
en el mercado de trabajo, ya que, en 2005, del total de los trabajadores, 35.7 por ciento era mujer y para 2015 esa proporción se incrementó a 37.7 por ciento, lo que significa que en una década creció en alrededor de 5.6 por ciento.

Ahora bien, revisando la situación laboral de los trabajadores, la mayoría se ubica como subordinado y remunerado. Del año 2005 al 2015 su proporción se incrementó, alcanzando un total de 67.8 por ciento. El segundo lugar es ocupado por aquellos que trabajan por cuenta propia; a diferencia del grupo anterior, se observó un decremento en su participación, pasando de 23.3 por ciento en 2005 a 22.4 por ciento en 2015. La participación de los que son patrones también decreció durante el periodo, y por tanto continúa siendo baja, dado que en 2015 solamente representó un poco más del cuatro por ciento del total de los trabajadores en el país. Este resultado nos señala que la gran mayoría de los trabajadores en México son asalariados. Sobre esta población se realizará el análisis de los datos y la inferencia estadística.

En la precariedad laboral, un aspecto clave son las prestaciones; de acuerdo a datos de la ENOE, en México se identifican principalmente cinco tipos de prestaciones las cuales se asocian fuertemente con el nivel de bienestar de la población: 1) vacaciones pagadas; 2) aguinaldo; 3) reparto de utilidades; 4) servicio médico; y 5) ahorro para el retiro. De estas cinco prestaciones, la proporción de trabajadores que perciben, servicio médico y ahorro para el retiro ha disminuido; y el resto creció por debajo del incremento de la población ocupada; es decir, ninguna prestación aumentó más allá de 2.2 puntos porcentuales que se incrementó la población ocupada; por tanto, estos movimientos que se observan durante una década fueron marginales.

Los porcentajes de la población que reciben las prestaciones se realizaron considerando únicamente a los trabajadores subordinados y remunerados. Con base a esta población, en 2015, 47.6 por ciento de los trabajadores no reciben vacaciones pagadas; 39.6 por ciento no recibe aguinaldo; 46.3 no cuenta con servicio médico; 53.8 no cuenta con ahorro para el retiro y lo más destacado es que 83.3 por ciento de los trabajadores no cuenta con reparto de utilidades. Esto significa que aquellos que se encuentran sujetos a un empleador, que como se mencionó previamente representan 67.8 por ciento de los trabajadores en 2015, una gran proporción no cuenta con estas prestaciones esenciales, y lo más preocupante es que la proporción de la población que no percibe estas prestaciones tiende a crecer.

La proporción de trabajadores que percibe prestaciones es baja, y todo parece indicar que para los siguientes años tenderá a disminuir, por lo que se espera que el tema de la precariedad laboral sea más relevante. Sin embargo, otros aspectos indicativos de precariedad son las jornadas de trabajo. La duración de la jornada donde la mayoría de los trabajadores subordinados y remunerados se ubican es de 48 horas por semana; sin embargo, de 2005 a 2015 ha disminuido esa proporción, pasando de 73.6 por ciento a 71.7 por ciento. En el caso de jornadas de trabajo superiores a 48 horas, la proporción de trabajadores aumenta, particularmente en jornadas superiores a 57 horas por semana. Este resultado implica que en una década una mayor proporción de trabajadores realizará jornadas de trabajo más extensas, afectando el tiempo que los trabajadores dedican a actividades de ocio. Las jornadas más largas las desarrollan principalmente los hombres. Cuando las jornadas son cortas la proporción entre hombres y las mujeres tiende a ser más equitativa, lo que significa que las mujeres tienden a desarrollar jornadas más cortas que los hombres.

Por otro lado, el nivel educativo en México ha mejorado, dado que hoy existe población con preparación profesional en una mayor proporción que hace una década. En el año 2005, el nivel primaria y se- 
cundaria predominaba entre los trabajadores, mientras que para 2015 predominaban los niveles secundaria, preparatoria y profesional. En estos dos últimos niveles educativos se ubica una gran parte de los trabajadores subordinados y remunerados, y son de los que mayor crecimiento han tenido en la última década. La proporción de trabajadores que cuenta con nivel de posgrado ha mejorado, aunque su participación continúa siendo baja. Lo que expone una mejora en la preparación académica de los trabajadores en México. Sin embargo, desde el punto de vista de Piketty (2014), la mejora en educación, no resulta un componente suficiente para explicar las diferencias salariales, al menos no en los países desarrollados.

Asimismo, el fenómeno de la precariedad se ha asociado frecuentemente a ciertas actividades económicas, sobre todo aquellas que no requieren personal con altos niveles de calificación, que fundamentalmente son las menos intensivas en capital. Con respecto a 2015, la industria manufacturera es considerada como una actividad económica intensiva en capital y por ello se esperaría que genere mejores condiciones laborales para los trabajadores; no obstante, en una década ha caído la proporción de trabajadores que labora en esta actividad. Para este mismo año, las actividades de gobierno, los servicios educativos, preparación de alimentos y bebidas y el comercio al por menor son actividades económicas que agruparon más de una tercera parte de los trabajadores en México. Estas actividades no son necesariamente actividades altamente productivas e intensivas en capital (según la clasificación de productividad de la OCDE, 2016), son más intensivas en mano de obra y con un perfil laboral de baja calificación $y$, por tanto, no se espera que los trabajadores que laboran en estas actividades cuenten con mejores condiciones de empleo. Tanto los servicios como las actividades primarias, en conjunto aumentaron en esta década, mientras que la manufactura y la construcción cayeron. Esto significa que, en una década, una mayor proporción de trabajadores labora en sectores de baja productividad. En este sentido, será necesario revisar el nivel de ingresos y su relación con cada actividad con el objeto de establecer el papel que juega cada sector en la generación de empleo precario.

Analizando el comportamiento del ingreso real mensual, el cual se estructuró con base al rango de salarios mínimos, tanto para 2005 como para 2015, se destaca el hecho de que la proporción de trabajadores que percibe ingresos entre uno y dos salarios mínimos, al igual de los que perciben entre dos y tres salarios, ha aumentado entre este periodo de análisis, y por el contrario disminuyó la proporción de trabajadores que perciben entre tres y cinco, y más de cinco salarios. Aunque este último resultado debe ser tomado con cautela, ya que para 2015 la proporción de trabajadores que no especificaron el nivel de ingresos es muy alta, seguramente una parte proporcional se deba a una menor proporción de trabajadores que perciben ese nivel de ingresos, pero cabe la posibilidad de que una proporción de los trabajadores de más de tres salarios, por cuestiones de seguridad, consideraron no pertinente declarar su rango de ingresos. También se observa una mejora en cuanto a la proporción de trabajadores que perciben hasta un salario mínimo, ya que entre 2005 y 2015 esa proporción disminuyó. En general, predominan los trabajadores que perciben hasta dos salarios mínimos, ya que representan 40 por ciento del total de la población que labora de forma subordinada o remunerada.

En relación al ingreso, es interesante revisar cómo se distribuye de acuerdo a los grupos de edad, con el propósito de descartar que se concentre en ciertos grupos de la población. El rango de más bajos ingresos, es decir, hasta dos salarios mínimos, es ocupado principalmente por los jóvenes entre 14 y 19 años y los adultos mayores. Sin embargo, se observa que entre mayor edad se tenga, disminuye el porcentaje de la población que se ubica en ese nivel de ingresos, esto es así hasta los 50-59 años, a partir de esta edad 
se vuelve a incrementar la proporción de la población que recibe el nivel de ingresos más bajo, por esta razón es que la proporción de la población de adultos mayores que recibe hasta dos salarios mínimos es alta. Tanto en 2005 como en 2015, la mayoría de los menores de 29 años percibían ingresos inferiores a los dos salarios mínimos; de los 30 años a los 39 la mayoría de los trabajadores percibe entre dos y tres salarios mínimos; y, para los de 40-49 el comportamiento fue variante: en 2005 predominaban los que percibían entre tres y cinco salarios y para 2015 la mayoría de los trabajadores en ese grupo de edad percibía entre uno y hasta cinco salarios mínimos. Y finalmente la mayoría de los trabajadores de más de 50 años, se ubicaba entre uno y dos salarios mínimos.

Otro aspecto interesante con respecto al ingreso y la edad, es que para la mayoría de los dos primeros rangos de ingresos se observa un incremento en su participación entre 2005 y 2015; y, por el contrario, para la mayoría de los niveles de ingresos superiores a los dos salarios mínimos disminuyó. En el caso de los que reciben más de cinco salarios mínimos, el resultado que se obtiene refleja que es poca la proporción de trabajadores que se colocan en ese nivel de ingresos.

Sin embargo, los bajos ingresos podrían estar asociados a jornadas de trabajo más cortas. Revisando el rango de ingreso de hasta un salario mínimo se observa que la mayoría de los trabajadores que percibe este nivel de ingresos realiza jornadas semanales de trabajo hasta 40 horas, y durante el periodo se observa que crece de manera significativa para este nivel de ingresos. En el caso de salarios de uno y hasta tres salarios, la mayoría de los trabajadores realizan jornadas de 41 a 48 horas a la semana, y en segundo lugar realizan jornadas hasta 40 horas, pero en ambos casos se observa una disminución entre 2005 y 2015, y una mayor proporción de trabajadores laborando en jornadas superiores a las 49 horas por semana. En el caso de salarios superiores a tres salarios mínimos, predominan jornadas hasta 40 horas, pero esta proporción de trabajadores ha disminuido y se han trasladado a jornadas de trabajo superiores. Es decir, hay un comportamiento creciente en la proporción de trabajadores que realizan mayores jornadas de trabajo, las cuales no necesariamente se asocian con mayores niveles de ingresos, sobre todo en la proporción de trabajadores que recibe entre dos y cinco salarios mínimos con jornadas mayores a las 57 horas semanales. Entonces, existe una mayor proporción de trabajadores que destina más horas a trabajar para percibir el mismo nivel de ingreso que percibía en 2005.

El comportamiento en la distribución de los salarios puede estar vinculado directamente con el nivel educativo de los trabajadores. En el caso del nivel de ingreso más bajo que corresponde hasta un salario mínimo, en la medida en que aumenta el nivel académico de los trabajadores su proporción va disminuyendo, lo que en principio concuerda con el hecho de que los menores ingresos se asocian a bajos niveles educativos. Un mayor nivel educativo en los trabajadores los traslada principalmente al rango de ingresos entre tres y más de cinco salarios mínimos. Los trabajadores con nivel medio superior, se ubican principalmente entre dos y hasta tres salarios mínimos; para nivel superior en 2005 predominaba la proporción que percibía más de cinco salarios y en 2015 predominaban los de salarios de tres y hasta cinco salarios mínimos. En el caso del nivel posgrado, la mayoría percibe más de cinco salarios mínimos, pero entre 2005 y 2015 esa proporción de trabajadores con ese nivel de estudios ha disminuido significativamente. A pesar de que con el nivel académico mejora el nivel de ingresos, cuando se revisa el cambio en las proporciones de 2005 a 2015, se encuentra que, en la mayoría de los casos, ha disminuido la proporción de trabajadores que percibían los salarios más altos en 2005 y que contaban con los niveles académicos de nivel medio superior, superior y posgrado, por lo que existe una tendencia a precarizar los 
niveles de ingreso de los trabajadores, aun cuando cuente con mayores niveles de educación.

Finalmente, se establece el papel que juega las principales actividades económicas que emplean a más de 76 por ciento de los trabajadores del país en relación con el ingreso. En las actividades menos productivas, la proporción de trabajadores tiende a ubicarse en su mayoría en rango de ingresos bajos, es decir, hasta dos salarios mínimos, tal es el caso de las actividades primarias, comercio al por mayor, servicios de hospedaje y otros servicios. Pero también, en este nivel de ingresos bajos se encuentra las manufacturas, que son consideradas actividades más productivas debido a que son más intensivas en capital, lo cual es de llamar la atención; sin embargo, entre 2005 y 2015 su proporción disminuyó. En el caso de trabajadores de servicios educativos y actividades gubernamentales, la mayoría percibe ingresos de tres y hasta cinco salarios mínimos. Por tanto, en todas las actividades productivas ha disminuido la proporción de trabajadores que percibe ese nivel de ingresos. En el nivel de ingresos de más de tres y hasta cinco salarios mínimos, la proporción de trabajadores ha disminuido, con excepción de las actividades primarias y la construcción. Este panorama expone una situación de mayor precariedad que se observa en todas las actividades económicas, y no solamente son producto de aquellos que desarrollan actividades de baja calificación.

Bajo esta primera revisión de datos estadísticos de la economía mexicana, se logra identificar que las condiciones laborales en México se han precarizado en la última década, por lo que resulta clave, como primer punto, crear una medida que logre englobar las características laborales que den cuenta del grado de precariedad que existe en México, e inferir cuál ha sido el rol de la educación, el sector, el tamaño de la empresa, el sexo, la edad y el estado civil, en la precariedad laboral que han experimentado los trabajadores en la última década.

\section{La construcción de la base de datos, variables y modelo}

Para dimensionar y estimar el fenómeno de la precariedad laboral en México por el lado de la demanda y oferta del mercado de trabajo, se utilizaron datos de la Encuesta Nacional de Ocupación y Empleo de 2005 y 2015. En este sentido, se pretende construir una medida de precariedad laboral y además ponderar los impactos que tienen tanto los aspectos socioeconómicos y sociodemográficos en dicha precariedad.

La variable precariedad laboral se construyó considerando las tres dimensiones que desde una visión de Román (2013) contextualizan dicha precariedad; el aspecto relacionado con el ingreso; la seguridad social; y el aspecto normativo, el cual lo asociaremos a la jornada de trabajo. Aunque desde el punto de vista normativo, es factible emplear otras variables, como aquella relacionada al contrato; sin embargo, dado que son variables que presenta una estructura multinivel; es decir, para identificar la precariedad laboral, resulta relevante si cuentan o no con contrato, y al mismo tiempo, el tipo de contrato; en este sentido, se prefirió emplear una sola variable, que por su propia estructura podría dimensionar el fenómeno de la precariedad, por lo que se consideró que para propósitos de construcción del modelo resultaba más apropiada la variable de jornada de trabajo.

La variable de precariedad laboral se medirá de manera ordinal, tomando los valores de 1, 2, 3 y 4, los cuales representan nada de precariedad, baja precariedad, media precariedad y alta precariedad, 
respectivamente. Para la construcción de cada nivel de precariedad, se tomaron en consideración tres condiciones del empleo con base en las dimensiones de Román (2013) que se mencionaron previamente: número de salarios mínimos, prestaciones laborales y jornadas de trabajo. En el caso del valor 1 referente a nada de precariedad se consideró que el trabajador: percibiera más de 6 salarios mínimos; en el tema de prestaciones contara con aguinaldo, vacaciones con goce de sueldo, reparto de utilidades, fondo de retiro y servicio médico; y que la jornada laboral fuera hasta 40 horas a la semana. Para el valor 2 que se vincula con baja precariedad: el número salarios que perciben es entre 4.1 y 6 salarios mínimos; en relación a las prestaciones este difiere con el anterior en que no recibe reparto de utilidades; la jornada de trabajo se determinó entre 41 y 50 horas a la semana.

Para el nivel de media precariedad, representado por el valor 3 se tomaron en cuenta que el trabajador: percibiera entre 2 y 4 salarios mínimos; las prestaciones que recibe son servicio médico, fondo de retiro y reparto de utilidades; y su jornada se definió entre 51 y 59 horas a la semana. Finalmente, para el nivel de precariedad alto, se tomaron en cuenta las siguientes condiciones: percepciones menores a los 2 salarios mínimos; ninguna prestación; y jornadas de trabajo superiores a las 60 horas a la semana.

Las variables que se consideraron en el estudio para determinar su impacto en la precariedad fueron: el nivel educativo, sexo, el estado civil, la actividad económica en donde labora, tamaño de la empresa y la edad de los trabajadores. La educación se utilizó en niveles y no en años de escolaridad, debido a que la escala de esta última para un mismo nivel de estudio se asociaba a diferentes niveles de precariedad, lo que genera inconsistencia en el modelo. A partir de estas variables, se pretender explicar bajo qué condiciones se ha presentado el fenómeno de la precariedad en el empleo en México. La relación técnica se plantearía de la siguiente manera:

$$
\operatorname{prelab}_{i}=f\left(\text { educación }_{i}, \text { sexo }_{i}, \text { estadocivil }_{i}, \text { sector }_{i}, \text { Tamaño }_{i}, \text { Edad }_{i}\right)
$$

donde

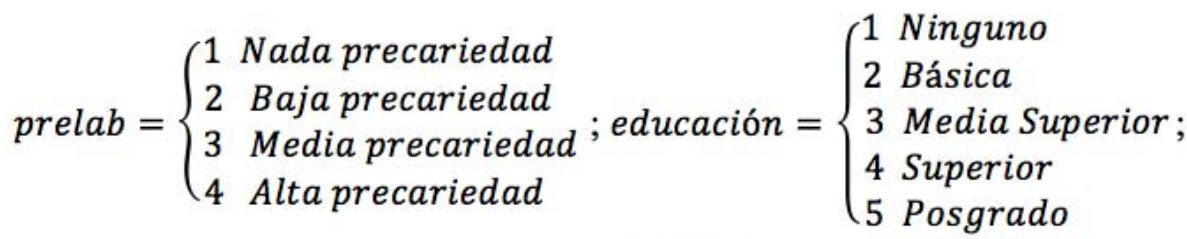

$$
\begin{aligned}
& \text { Sexo }=\left\{\begin{array}{l}
0 \text { Hombre } \\
1 \text { Mujer }
\end{array} ;\right. \\
& \text { EstadoCivil }=\left\{\begin{array}{ll}
0 & \text { No unido } \\
1 & \text { Unido }
\end{array} ; \text { Sector }=\left\{\begin{array}{ll}
1 & \text { Construcción } \\
2 & \text { Industria Manufacturera } \\
3 & \text { Comercio } \\
4 & \text { Servicios } \\
5 & \text { Otros } \\
6 & \text { Agropecuario }
\end{array} ;\right.\right.
\end{aligned}
$$




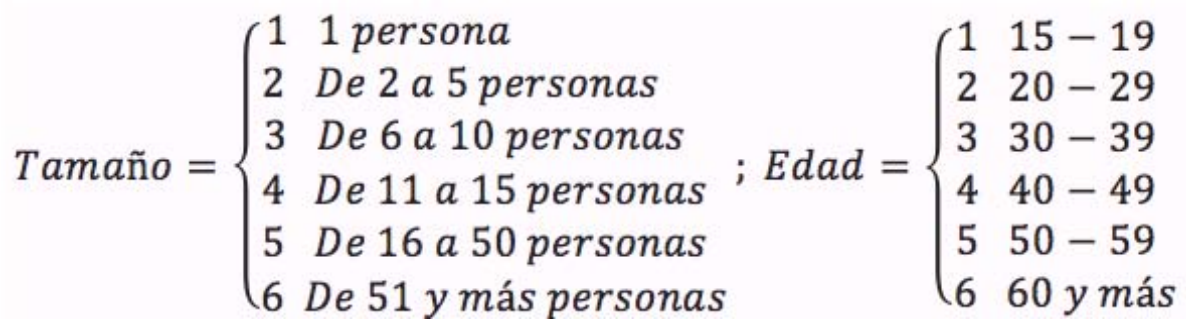

Para estimar la relación, dada la naturaleza de la construcción de la variable dependiente, cuya estructura se asocia al modelo de carácter ordinal, la estimación econométrica de la relación se realizó con base en el modelo logístico ordinal. Aunque existen otros modelos logísticos como el caso del tipo multinomial; sin embargo, en este modelo el orden que adopta la variable dependiente no importa. Caso contrario sucede en el modelo logístico ordinal, en donde la probabilidad de adoptar un valor específico o superior, se asocia a la probabilidad de un conjunto de valores que pueden ser iguales y menores a dicho valor (Liu, 2016). Este tipo de técnicas han sido ampliamente utilizadas en ámbito de la salud, por ejemplo, para medir estrés; en el ámbito comercial para medir la satisfacción; en lo social para dimensionar percepción de inseguridad, entre otras que son expuestas en Liu (2016). Utilizar este modelo ordinal que ha sido implementado en diferentes campos del conocimiento, para dimensionar el fenómeno de la precariedad en México, resulta un reto para el presente trabajo de investigación.

Las probabilidades de ocurrencia del modelo ordinal se asocian a los valores que adopta la variable dependiente y pueden expresarse de la siguiente manera:

$$
\theta_{j}=\frac{P(\text { valor } \leq j)}{P(\text { valor }>j)}=\frac{P(\text { valor } \leq j)}{(1-P(\text { valor } \leq j))}
$$

donde puede adoptar los valores de 1, 2, 3 y 4, correspondientes a los diferentes niveles de precariedad que se mencionaron previamente. Si bien estas escalas de medición no describen de manera precisa el fenómeno de la precariedad, sí se aproximan a su entendimiento, por lo que se clasifican en la familia de variables latentes.

El modelo Logístico Ordinal se expresa de la siguiente manera:

$$
\ln \left(Y_{j}\right)=\operatorname{logit}[\Gamma(x)]=\ln \left(\frac{\Gamma(x)}{1-\Gamma(x)}\right)=\rho_{j}+\left(-\phi_{1} X_{1}-\phi_{2} X_{2}-\cdots-\phi_{p} X_{p}\right)
$$

donde representa la probabilidad acumulada de localizarse en o por debajo de la categoría j; es el punto de corte asociado a los distintos valores y son los coeficientes logísticos que determinan los impactos de las variables explicativas sobre la variable explicada. Para las diversas categorías de la variable dependiente, el modelo de probabilidades proporcionales estima punto de cortes. Entonces el modelo Logístico Ordinal para los diferentes valores de se puede representar de la siguiente manera:

$$
\begin{gathered}
\ln \left(Y_{j}\right)=\operatorname{logit}\left[\Gamma\left(Y \leq j \mid x_{1}, x_{2}, \ldots, x_{p}\right)\right]=\ln \left(\frac{\Gamma\left(Y \leq j \mid x_{1}, x_{2}, \ldots, x_{p}\right)}{\Gamma\left(Y>j \mid x_{1}, x_{2}, \ldots, x_{p}\right)}\right)=\rho_{j}+\left(-\phi_{1} X_{1}-\phi_{2} X_{2}-\right. \\
\left.\cdots-\phi_{p} X_{p}\right)
\end{gathered}
$$


Este modelo econométrico asume que los impactos de los predictores serán los mismos a través de los distintos valores de la variable dependiente ordinal, que este caso es la precariedad en el empleo, este supuesto se conoce con el nombre probabilidades proporcionales o líneas paralelas (Ari y Yildiz, 2014; Kleinbaum y Klein, 2010; Liu, 2016). Este supuesto generalmente no se cumple en estimaciones ordinales, principalmente debido al tamaño de muestra y el número de patrones covariantes. Si este supuesto no se cumple, la estimación del modelo Logístico Ordinal generará resultados e interpretaciones erróneas. El método alternativo en ausencia de líneas paralelas es el modelo Logístico Ordinal Generalizado, el cual relaja este supuesto permitiendo que los impactos de las variables independiente varíen a través de los distintos puntos de cortes de la variable dependiente (Liu y Koirala, 2012; Liu, 2016). Este modelo se expresa como:

$$
\begin{gathered}
\ln \left(Y_{j}\right)=\operatorname{logit}\left[\Gamma\left(Y>j \mid x_{1}, x_{2}, \ldots, x_{p}\right)\right]=\ln \left(\frac{\Gamma\left(Y>j \mid x_{1}, x_{2}, \ldots, x_{p}\right)}{\Gamma\left(Y \leq j \mid x_{1}, x_{2}, \ldots, x_{p}\right)}\right)=\rho_{j}+\left(-\phi_{1 j} X_{1}-\right. \\
\left.\phi_{2 j} X_{2}-\cdots-\phi_{p j} X_{p}\right)
\end{gathered}
$$

Este modelo estima las probabilidades de estar más allá de una cierta categoría, en este caso se hace alusión a las categorías de precariedad, en relación a estar en o por debajo de esa categoría. Un coeficiente positivo logit generalmente indica que un individuo es más probable que esté en una categoría de mayor nivel de precariedad en oposición a una categoría de menor precariedad. En este modelo, todos los impactos de las variables explicativas varían a través de cada uno de los puntos de cortes.

Para verificar el supuesto de líneas paralelas y definir el método correcto de estimación, se utilizará la prueba de Brant. De manera complementaria se revisarán los resultados de los criterios Bayesiano y Akaike para definir la pertinencia del Modelo Ordinal o del Modelo Ordinal Generalizado. Para analizar la pertinencia del modelo, se utilizaron los criterios estadísticos de Wald, su probabilidad, Pseudo , estadístico Z y su probabilidad. Los comandos empleados en el programa Stata versión 14 son ologit y gologit2.

Cabe hacer mención, que el análisis se realizó para la población subordinada mayores a 15 años de edad, a partir de la muestra que proporciona el INEGi sobre la Encuesta Nacional de Empleo de Ocupación y empleo de 2005 y 2015; sin embargo, se ampliaron a niveles poblaciones a través del factor de expansión que proporciona el mismo instituto.

\section{La precariedad del trabajo desde una visión de la demanda de trabajo: resultados econométricos}

Tanto la argumentación teórica como los resultados expuestos en apartados previos señalan que el trabajo en México se ha precarizado, se tienen jornadas más largas, se reducen los ingresos reales y disminuyen las prestaciones, lo que afecta el bienestar de los trabajadores. Integrando estas condiciones laborales de forma ordinal en la variable precariedad laboral, se puede observar de los resultados que se presentan en el Cuadro A1 y de los gráficos del I al VI, cómo la proporción de los trabajadores que experimentaron Nada y Baja precariedad entre 2005 y 2015 ha disminuido y, por el contrario, ha crecido la proporción de trabajadores que experimentan media y alta precariedad laboral. 
Esta mayor presencia de trabajadores en condiciones de precariedad se ha presentado sin distingo de sexo, estado civil, edad, nivel educativo, tamaño de la empresa e incluso sin distingo de sectores; esto es, la precariedad en el empleo se ha generalizado en el país, aunque de los resultados econométricos que se presentan en el Cuadro 3, se observa que las probabilidades de experimentar un mayor nivel de precariedad difieren entre cada una de estas variables.

Pero antes de revisar puntualmente los resultados econométricos, es importante mencionar que derivados de la prueba de Brant y de los resultados de las pruebas de Akaike y de Bayesiano, las cuales se presentan en los cuadros 1 y 2 , se encontró que el modelo Logístico Ordinal viola el supuesto de líneas paralelas tanto en 2005 como en 2015, y, por tanto, se empleó el método de estimación Logístico Ordinal Generalizado, el cual proporciona resultados más robustos. De esta manera, se estimaron los coeficientes del modelo que se presentan en el Cuadro 3 y los resultados gráficos que se muestran en el anexo, adicionalmente, en este anexo se presentan las probabilidades marginales de cada una de las variables para cada nivel de precariedad para los años de 2005 y 2015.

\begin{tabular}{|c|c|c|}
\hline \multicolumn{2}{|c|}{ Cuadro 1. Prueba Brant } \\
\hline \multicolumn{2}{|c|}{ Pariable explicada: Nivel de precariedad del empleo. } \\
\hline Variables explicativas & 2005 & 2015 \\
\hline Escolaridad & 0 & 0 \\
\hline Sexo & 0.082 & 0.869 \\
\hline Estado civil & 0.017 & 0.002 \\
\hline Sector & 0.016 & 0.006 \\
\hline Número de empleado & 0 & 0 \\
\hline Edad & 0 & 0.175 \\
\hline
\end{tabular}

Fuente: Elaboración propia con base en datos de la ENOE 2005 y 2015.

\section{Cuadro 2. Prueba de Akaike y Bayesiano para definir el método de estimación}

\begin{tabular}{|c|c|c|c|c|}
\hline \multirow{2}{*}{} & \multicolumn{2}{|c|}{2005} & \multicolumn{2}{c|}{2015} \\
\hline & Ologit & gologit & ologit & gologit \\
\hline AIC & 2344672.33 & 2292165.4 & 2549475.28 & 2506144.58 \\
\hline BIC & 2344782.58 & 2292422.65 & 2549586.39 & 2506403.83 \\
\hline
\end{tabular}

Fuente: Elaboración propia con base en datos de la enoe 2005 y 2015. 
Partiendo de los resultados que se presentan en el Cuadro 3, en relación al tema de la educación, se encuentra que continúa siendo la que influye en mayor medida en el grado de precariedad que pudieran experimentar los trabajadores. De los resultados de este cuadro, la educación es la que mayor impacto tiene como determinante de la precariedad laboral. Comparando el resultado de nada de precariedad con un mayor nivel de precariedad, se observa que en 2005 decrece la probabilidad de ubicarse en un mayor nivel de precariedad laboral cuando el nivel educativo mejora, en alrededor de 76 por ciento, mientras que en 2015 esta probabilidad fue de 68 por ciento. Cuando se comparan los dos restantes grados de precariedad en cuanto al tema educativo, se encuentra un resultado similar: entre mayor nivel educativo disminuye marginalmente la probabilidad de ubicarse en una categoría de mayor precariedad, como se observa en el cuadro A.2 del Anexo, con base en las probabilidades marginales, se identifica que aún en presencia de mayor educación, existe en 2015 una mayor probabilidad de estar en un nivel de precariedad bajo y alto en comparación a 2005; sin embargo, su impacto para 2015 en comparación con 2005 tiende a ser menor, lo cual se puede observar en el gráfico VI de 2005 y 2015, en donde la curva de nada de precariedad disminuyó para todos los grados académicos, y la curva de baja, media y alta precariedad se desplazó hacia arriba, principalmente en los niveles educativos superior y de posgrado. Lo que significa que aun cuando la educación representa un factor que mejora las condiciones del empleo en México, en la actualidad, su impacto ha sido menor, ya que los trabajadores de todos los niveles educativos experimentan un mayor grado de precariedad en el empleo. Pero, además, aunque los niveles educativos han mejorado en los países desarrollados y con ellos el nivel de vida de la población, esta mejora no ha reducido la desigualdad en el ingreso por trabajo (Piketty, 2014). En el caso de México, y aludiendo los resultados de Huesca y Ochoa (2016), es posible que el menor impacto de la educación en los salarios se deba a la menor presencia de inversión productiva de alta tecnología, que demande trabajadores con altos niveles educativos. De hecho, en su estudio señalan que la menor demanda de mano con altos niveles educativos es lo que ha provocado que la desigualdad salarial disminuya en México.

\begin{tabular}{|c|c|c|c|c|}
\hline \multicolumn{5}{|c|}{ Variable explicada: Nivel de precariedad del empleo. } \\
\hline \multirow[t]{2}{*}{$\begin{array}{l}\text { Variables expli- } \\
\text { cativas }\end{array}$} & \multicolumn{2}{|c|}{ Coeficientes } & \multicolumn{2}{|c|}{ Odd Ratio } \\
\hline & 2005 & 2015 & 2005 & 2015 \\
\hline \multicolumn{5}{|c|}{$N P>1$ vs $N P \leq 1$} \\
\hline Educación & -1.44 & -1.15 & $0.24^{*}$ & $0.32 *$ \\
\hline Sexo & -0.43 & -0.23 & $0.65^{*}$ & $0.79 *$ \\
\hline Estado civil & -0.25 & -0.38 & $0.78 *$ & $0.69 *$ \\
\hline Sector & 0.15 & 0.23 & $1.17^{*}$ & $1.26^{*}$ \\
\hline Empleados & -0.57 & -0.81 & $0.57^{*}$ & $0.44^{*}$ \\
\hline Edad & -0.44 & -0.26 & $0.64 *$ & $0.77^{*}$ \\
\hline Constante & 10.78 & 10.7 & 48085.88* & 44294.27* \\
\hline
\end{tabular}




\begin{tabular}{|c|c|c|c|c|}
\hline \multicolumn{5}{|c|}{$N P>2$ vs $N P \leq 2$} \\
\hline Educación & -1.16 & -1.2 & $0.31 *$ & $0.30 *$ \\
\hline Sexo & -0.11 & -0.17 & $0.89 *$ & $0.84 *$ \\
\hline Estado civil & -0.53 & -0.62 & $0.59 *$ & $0.54^{*}$ \\
\hline Sector & 0.2 & 0.21 & $1.22 *$ & $1.23 *$ \\
\hline Empleados & -1.02 & -1.1 & $0.36 *$ & $0.33^{*}$ \\
\hline Edad & -0.23 & -0.28 & $0.79 *$ & $0.76^{*}$ \\
\hline Constante & 7.79 & 8.84 & 2417.1* & 6917.41* \\
\hline \multicolumn{5}{|c|}{$N P>3$ vs $N P \leq 3$} \\
\hline Educación & -1.2 & -1.04 & $0.30 *$ & $0.35 *$ \\
\hline Sexo & -0.02 & -0.15 & $0.98 *$ & $0.86^{*}$ \\
\hline Estado civil & -0.45 & -0.52 & $0.64 *$ & $0.60 *$ \\
\hline Sector & 0.2 & 0.28 & $1.22 *$ & $1.32 *$ \\
\hline Empleados & -0.87 & -0.91 & $0.42 *$ & $0.40 *$ \\
\hline Edad & -0.22 & -0.25 & $0.80 *$ & $0.78^{*}$ \\
\hline Constante & 6.45 & 6.47 & $629.69 *$ & $645.46^{*}$ \\
\hline $\mathbf{N}$ & $1,544,194$ & $1,699,047$ & $1,544,194$ & $1,699,047$ \\
\hline Wald chi2(18) & 899585.33 & 9600084.59 & 899585.33 & 9600084.59 \\
\hline Prob (Chi2) & 0 & 0 & 0 & 0 \\
\hline Pseudo R2 & 0.3683 & 0.3715 & 0.3683 & 0.3715 \\
\hline
\end{tabular}

Fuente: Elaboración propia con base en datos de la enoe 2005 y 2015. * significativos al 95 por ciento de confianza.

El segundo factor en importancia que determina el grado de precariedad es el tamaño de la empresa, el cual se midió por el número de empleados en rangos. Para la categoría de nada de precariedad, en relación con un mayor grado de precariedad se observa que en 2005 entre más grande la empresa disminuyó 43 por ciento la probabilidad de ubicarse en un nivel de mayor precariedad; para 2015 esta probabilidad se incrementó, alcanzando una probabilidad de 56 por ciento. Esta misma situación se observa para las dos subsecuentes categorías de precariedad. Por lo que denota que, entre 2005 y 2015, el tamaño de la empresa ha sido uno de los principales determinantes para dimensionar el fenómeno de la precariedad en el trabajo, conjuntamente con el nivel educativo, de hecho, de acuerdo al Cuadro A.2, se aprecia que en ambas crece más la probabilidad tanto en baja como en alta precariedad entre 2005 y 2015. Particularmente, las empresas con pocos trabajadores son las que tienden a contratar trabajadores en condiciones más precarias, aunque las empresas con mayores empleados tienden a presentar baja precariedad; es decir, con bajos salarios, jornadas de trabajo largas y menores prestaciones laborales, principalmente debido a que en este tipo de establecimientos se carece de regulación en las relaciones laborales principalmente porque tienden a predominar contratos individuales sin respaldo sindical, por el escaso personal que existe en el establecimiento (Solís, 2014). Sin embargo, Samaniego (2010) señala que también en los grandes establecimientos muestran una tendencia a elevar el número de trabajadores en condiciones de precariedad. 
El resto de los determinantes de la precariedad tienen resultados diferentes de acuerdo al nivel de precariedad que se compare. Respecto a la edad, cuando se compara la categoría de nada de precariedad con un mayor nivel de precariedad, este ocupa el tercer lugar en importancia, y lo que permite identificar el resultado es que a mayor edad disminuye la probabilidad de una mayor precariedad laboral; esto es, para 2005 un año más de edad en el trabajo disminuye su probabilidad en 36 por ciento de experimentar una mayor precariedad en el empleo, pero esa proporción disminuyó para 2015, siendo solamente de 24 por ciento. Y conforme se experimenta más precariedad, que es el caso de las dos categorías restantes, el impacto de la edad sobre la posibilidad de experimentar un mayor nivel de precariedad disminuye; es decir, cuando la población ya experimenta precariedad, es posible asumir que pueda experimentar aun mayor nivel precariedad. Al revisar la probabilidad marginal en el Cuadro A.2, se destacan los resultados de nada y baja precariedad, en donde en el primer caso a pesar de ser positiva, disminuye la probabilidad de 2005 a 2015, mientras que, en la segunda, aumenta la probabilidad en ese mismo periodo, corroborando que en dicho periodo se transita hacia un escenario de mayor precariedad.

Observando la gráfica 3a para ambos años, los jóvenes son lo que mayor probabilidad tienen de experimentar un mayor nivel de precariedad laboral; sin embargo, entre 2005 y 2015, se incrementó la probabilidad de la curva de alta precariedad, ya que para todos los grupos de edad se observan mayores probabilidades. En 2005, la probabilidad de que los jóvenes de 15 a 19 años tuvieran un alto nivel de precariedad era de 43.4 por ciento, en 2015 se elevó a 50.5 por ciento. Aunque todos los grupos etarios experimentan precariedad, en el caso de los jóvenes, han sido los más vulnerados al adoptar involuntariamente modelos de contratación como el "outsourcing" y la subcontratación que se han ido construyendo desde los inicios del modelo maquilador en México, y reconocidos legalmente desde la aprobación de la última reforma laboral de 2012, con el único propósito de disminuir los costos laborales y elevar las ganancias de los empresarios (Anguiano y Ortiz, 2013).

En el caso del grupo de edad de 60 y más se observó que pasó de 20.2 a 22.5 por ciento durante ese mismo periodo. Caso contrario con la curva de nada de precariedad, donde se observó que su probabilidad disminuyó para todos los casos. En el caso de las edades de 15 a 19 se tenía en 2005 una probabilidad de 1.7 por ciento y para 2015 fue de 1.4 por ciento, mientras que en el caso de los adultos mayores en 2005 se tenía una probabilidad de 14.6 por ciento y en 2015 se registró una probabilidad de 5 por ciento y disminuyó significativamente la probabilidad de que los adultos mayores experimentaran un nivel de precariedad superior.

Este fenómeno de precariedad entre 2005 y 2015 se ha acentuado principalmente en el terreno de los hombres, ya que de los resultados del cuadro de estimación se observa que la probabilidad de experimentar una mayor precariedad es mayor para los hombres, mientras que para las mujeres disminuye dicha probabilidad. No obstante, el impacto entre ambos periodos ha disminuido, debido que tanto en los hombres como en las mujeres ha crecido la proporción de un mayor nivel de precariedad laboral, aunque los impactos para los hombres continúan siendo mayores. Revisando los estadísticos del Cuadro 3 , se puede apreciar que el crecimiento en la proporción de hombres que puede caer en una condiciDe igual manera, al revisar el grafico ral, aunque los impactos para los hombres continua siendo mayor, es ha crecido la proporcón de precariedad es mayor que en el caso de las mujeres, marginalmente a lo largo de las distintas categorías analizadas. De igual manera, al revisar el gráfico I del anexo, la probabilidad de experimentar un mayor grado de precariedad es mayor para los hombres en ambos años; sin embargo, se 
ha ampliado la diferencia, lo que corrobora que en los hombres se observa un ligero aumento en el nivel de precariedad laboral. Aunque analizando la probabilidad marginal del Cuadro A.2, esta diferencia se acentúa más en el caso de alta precariedad.

En lo que respecta al tema del estado civil, los resultados muestran que es menos probable que se presenten en los unidos. La primera categoría de nada de precariedad contra un nivel de precariedad, se obtuvo en 2005 que para los unidos disminuye en 22 por ciento la probabilidad de caer en un mayor nivel de precariedad laboral, mientras que en 2015 esta probabilidad se ubicó en 31 por ciento. En los siguientes dos casos esas probabilidades son mayores y crecen de 2005 a 2015, lo que implica que es un fenómeno que tiende a presentarse, en mayor medida, en poblaciones con un estado civil de no unidos. De hecho, en los estadísticos del cuadro se observa cómo la proporción de media y alta precariedad de los trabajadores no unidos es mayor, aunque en ambos crece la proporción de un periodo a otro, situación que se puede apreciar con mayor precisión en el gráfico II, en donde la alta precariedad crece tanto para los unidos como para los no unidos, aunque para este último grupo su crecimiento fue mayor. Considerando la probabilidad marginal del cuadro A.2, se observa que, en el caso de la alta precariedad, resulta ser mayor el impacto negativo en 2015 que en 2005, por lo que la probabilidad de los unidos de estar en una peor situación laboral es menor que en relación a los no unidos.

Finalmente, en relación a los sectores, los resultados muestran una relación directa entre el sector (ordenado de mayor a baja productividad) y el nivel de precariedad laboral. Esto significa que los trabajadores que laboran en sectores menor productivos tienden a presentar un mayor grado de precariedad. En la primera categoría donde se compara nada de precariedad con un mayor nivel de precariedad se encuentra en 2005 que laborar en sectores menos productivos incrementa en 17 por ciento la probabilidad de experimentar una mayor precariedad, mientras que en 2015 es de 26 por ciento de probabilidad. Para las siguientes categorías que denotan un mayor nivel de precariedad, se observa que la probabilidad se incrementa tanto en 2005 como en 2015, aunque nuevamente resulta ser más alta en 2015, lo que significa que entre mayor precariedad laboral se experimente es más probable que esta se incremente cuando se labora en sectores menos productivos, como se corrobora en el cuadro A.2. donde la probabilidad marginal crece de 2005 a 2015 para el caso de la precariedad alta.

Del cuadro de estadísticos A1 del anexo, se aprecia como la mayor proporción de trabajadores en la categoría de nada de precariedad y baja precariedad se ubica en el sector construcción y manufactura, mientras que en la categoría de alta precariedad la mayor proporción labora en el sector servicios y agropecuarios. Sin embargo, entre 2005 y 2015, todos los sectores disminuyeron la proporción de trabajadores que laboraban en nada y baja precariedad. En el caso de la media precariedad, el único sector que no aumentó su proporción fue el agropecuario. Mientras que, en la categoría de alta precariedad, el único sector que no incrementó su proporción fue el sector construcción. Al comparar entre 2005 y 2015 la pendiente de alta precariedad, en el gráfico VI se observa que esta tiende a ser mayor en 2015, lo que expone, que independientemente del sector que se labore, todos experimentaron una mayor proporción de precariedad. Esta precariedad generalizada que se presenta hasta en sectores de alta productividad coincide por lo argumentado por Anguiano y Ortiz (2013) y por Mora-Salas y Oliveira (2009), quienes puntualizan que este fenómeno de hacer más competitivas a las empresas se ha realizado a expensas de la explotación laboral, y que se ha generalizado a todos los sectores productivos de la economía, incluso al mismo gobierno. 
Estos indicadores y estimaciones de las condiciones del empleo, asociadas a características socioeconómicas y sociodemográficas, presentan un escenario de una creciente precariedad en el empleo en México en una década, que desde una visión de Solís (2014) no solo se trata de bajos ingresos, jornadas más largas y menores prestaciones, sino que también contiene un componente subjetivo que se asocia a un sentimiento de inseguridad de poder contar con los recursos necesarios para cubrir necesidades básicas, tales como las que señala Tomasina (2012), la educación, alimentación, vivienda, entre otros, que pudieran tener un efecto negativo en la salud del trabajador, a través de la manifestación de nerviosismo, ansiedad, depresión, temor, problemas para socializar, entre otras afectaciones colaterales que podrían surgir de la presencia de una mayor precariedad en el empleo.

\section{Conclusión}

La precariedad del trabajo ha tomado mayor relevancia en los últimos años, dado que el fenómeno ha crecido de manera sostenida en el mundo. Existe el consenso internacional de que esta situación surge como consecuencia de la liberalización de la economía y la apertura comercial, ya que, para promover mercados más competitivos y menos vulnerables a entornos de crisis y recesiones económicas, se modificaron los mecanismos de contratación del personal, haciendo flexible el mercado de trabajo. Esta flexibilización se ha impulsado bajo el argumento que favorece la competitividad de las empresas, ya que al reducir los costos laborales se incrementa la rentabilidad de las empresas; además, las hace menos vulnerables a los ciclos económicos. Otro aspecto que resalta de esta flexibilización que ha predominado en el mundo del trabajo es que, en épocas de bonanza económica, beneficia a los trabajadores, ya que se generan más empleos. Sin embargo, lo que en realidad se observa es que con la flexibilidad laboral se han generado efectos negativos que particularmente se han trasladado a los trabajadores, ya que el valor que se le asigna al trabajo que realizan ha disminuido, exponiéndolos a un contexto precario y sin estabilidad laboral, quedando expuestos a crisis y recesiones económicas. Así, el empresario ha transitado a un esquema de subcontratación de trabajadores; es decir, renta trabajadores, lo que le ha permitido elevar la rentabilidad de la empresa y afectar el nivel de vida de los trabajadores (Piketty, 2014).

Del análisis teórico y de la construcción de información descriptiva de la Encuesta Nacional de Ocupación y Empleo 2005 y 2015 sobre trabajadores subordinados y remunerados, se construyó una medida que permite identificar los diferentes grados de precariedad a la que se encuentran expuestos los trabajadores en México y a partir del Modelo Logístico Ordinal Generalizado, se identificó en qué medida el sexo, la edad, el sector económico, el nivel educativo, el estado civil y el tamaño del establecimiento contribuyen en la precariedad del empleo en México durante ese periodo. La variable precariedad laboral adoptó valores ordinales, tomando en consideración tres características: jornadas de trabajo, nivel de ingresos y prestaciones laborales.

Los resultados presentados apuntan que, en una década, la proporción de trabajadores que laboran en condiciones más precarias ha crecido; es decir, que el número de personas que reciben menos salarios, menores prestaciones y trabajan mayores jornadas es mayor. En el tema de las prestaciones, se encontró que ha disminuido la proporción de trabajadores que reciben servicios médicos y ahorro para el retiro, y que la proporción de los trabajadores que no reciben reparto de utilidades es muy alta, durante el periodo 
alcanzó alrededor de 83 por ciento de los trabajadores subordinados y remunerados del país. También ha aumentado la proporción de trabajadores que desarrollan jornadas de trabajo más extensas y no se corresponden con mayores ingresos reales; por el contrario, para poder mantener el mismo nivel de ingresos que hace 10 años los trabajadores tienen que dedicar mayor cantidad de horas a laborar.

El nivel educativo en México ha crecido en los últimos 10 años: en 2005 la mayoría de los trabajadores tenía el nivel primaria y secundaria, para 2015 la mayoría se ubicaba en un nivel secundaria, preparatoria y profesional. En estos dos últimos niveles educativos se ubican una gran parte de los trabajadores subordinados y remunerados, y son de los que mayor crecimiento se ha tenido en la última década. La proporción de trabajadores que cuenta con nivel de posgrado también ha mejorado. Los resultados del modelo econométrico muestran que la educación continúa siendo la que influye en mayor medida en el grado de precariedad que pudieran experimentar los trabajadores, pero en una década su impacto disminuyó, ya que los trabajadores de todos los niveles educativos experimentan un mayor grado de precariedad en el empleo. En este sentido, la precariedad laboral no puede ser explicada a partir del rezago educativo, más bien parece ser el resultado de las decisiones de las empresas por mejorar su condición de competitividad en los mercados. Por esta razón, los resultados econométricos señalan que la precariedad laboral ha aparecido en todos los sectores económicos y para todos los tamaños de las empresas, aunque esta sigue siendo mayor en sectores de baja productividad y en aquellos de menor tamaño, respectivamente. Sin embargo, la precariedad ha crecido de manera sistemática bajo estas condiciones.

En cuanto al tema de la edad, los resultados muestran que tanto los jóvenes como los adultos mayores son los dos grupos de la sociedad en donde la probabilidad de una mayor precariedad laboral ha crecido, aunque en todos los casos se encuentra mayor precariedad laboral entre 2005 y 2015 . Y son precisamente los hombres los que más afrontan esta situación de precariedad durante este periodo. En cuanto al estado civil, se encuentra que existe una mayor probabilidad de que los no unidos presenten mayor precariedad en relación con los unidos, lo que resulta consistente con el tema de la edad, ya que esta última condición prevalece en los jóvenes y en los adultos mayores.

En general, los resultados econométricos señalan que, en una década, la clase subordinada y remunerada ha incrementado su probabilidad de experimentar mayor precariedad laboral, sin importar el sector, el sexo, la educación, el tamaño del establecimiento, la edad o el estado civil. Aunque estas características socioeconómicas y sociodemográficas del mercado de trabajo continúan generando efectos diferentes en cuanto a los impactos de cada uno de ellas sobre el grado de precariedad que pudieran experimentar los trabajadores. Con estos resultados, queda de manifiesto que son elementos de la demanda de trabajo los que han deteriorado las condiciones de los trabajadores en México durante este periodo, ya que, a pesar de haber mejorado el nivel educativo de los trabajadores, la tendencia de precarizar el empleo continúa creciendo, sin importar el tamaño del establecimiento y el sector económico. Entonces, la precariedad ha sido producto de las decisiones de las empresas que, en su búsqueda por obtener mejores beneficios, han reducido los costos de producción, a través de reducir salarios y prestaciones y aumentar jornadas de trabajo en la clase trabajadora asalariada que, en México, se compone por 68 por ciento del total de la población que labora.

La precariedad laboral que afecta a los trabajadores y sus familias no solo se limita al tema de menores salarios, menores prestaciones y mayores jornadas de trabajo, que ya en sí son un problema social y económico, sus efectos podrían tener un alcance mayor al asociarse a problemas de salud pública, como 
estrés, depresión, nerviosismo, ansiedad, temor, problemas de socialización, entre otras afecciones. Es por ello que se requiere revertir este proceso de empobrecimiento laboral, reasignándole un mayor valor al trabajo a través de estrategias que haga más productivo al capital pero que al mismo tiempo puedan trasladar esa mayor rentabilidad a los trabajadores.

\section{Referencias}

Anguiano, Arturo y Rosario Ortiz. 2013. Reforma laboral en México: precarización generalizada del trabajo. El Cotidiano, 182: 95-104.

Aparicio, Pablo. 2013. Educar y trabajar en contextos de precariedad y desigualdad en América Latina. Jóvenes en debate. Revista Latinoamericana de Ciencias Sociales, Niñez y Juventud, 11(2): 527-546.

Ari, Erkan y Yildiz Zeki. 2014. Parallel lines assumption in Ordinal Logistic Regression and analysis approaches. International Interdisciplinary Journal of Scientific Research, 1(3): 8-23.

Barattini, Mariana. 2009. El trabajo precario en la era de la globalización. ¿Es posible la organización? Polis, Revista de la Universidad Bolivariana, 8 (24): 17-37.

Espinosa, Alejandro y Raúl Hernández. 2013. Precarización del trabajo y reestructuración productiva en México. El retorno del desarrollo en México. México, D. F.: UAM-X, CsH, Depto. de Producción Económica. Fernández, Mariana. 2014. Dimensiones de la precariedad laboral: un mapa de las características del empleo sectorial en la Argentina. Cuadernos de Economía, 33(62): 231-257. https://doi.org/10.15446/ cuad.econ.v33n62.43675

Huesca, Luis y Gloria Ochoa. 2016. Desigualdad salarial y cambio tecnológico en la Frontera Norte de México. Problemas del Desarrollo, 47(187): 165-188. https://doi.org/10.1016/j.rpd.2016.10.006

Instituto Nacional de Estadística, Geografía e Informática. 2005. Encuesta Nacional de Ocupación y Empleo (ENOE). México.

Instituto Nacional de Estadística, Geografía e Informática. 2015. Encuesta Nacional de Ocupación y Empleo (ENOE). México.

Julián, Dasten. 2014. Bases del modelo de valoración precario del trabajo en Chile. Acercamientos desde la política laboral y la cultura del trabajo. Sociológica, 29(81): 119-160.

Kleinbaum, David y Klein Mitchel. 2010. Logistic regression: A self-learning text. Springer. Nueva York. United States of America. https://doi.org/10.1007/978-1-4419-1742-3_1

Liu, Xing. 2016. Applied ordinal logistic regression using Stata: From single-level to multilevel modeling. Nueva York: SAGE Publications.

Liu, Xing y Hari Koirala. 2012. Ordinal regression analysis: Using generalized ordinal logistic regression models to estimate educational data. Journal of Modern Applied Statistical Methods, 11(1): 242-254.

Mora-Salas, Minor y Orlandina de Oliveira. 2009. La degradación del empleo asalariado en los albores del siglo XXI: Costa Rica y México. Papeles de Población, 15(61): 195-231.

OECD. 2016. OECD Compendium of productivity indicators 2016. Paris: OECD Publishing. http://dx.doi. org/10.1787/pdtvy-2016-en

Piketty, Thomas. 2014. El capitalismo en el siglo XXI. México: Fondo de Cultura Económica. 
Román, Yuliana. 2013. Impactos sociodemográficos y económicos en la precariedad laboral de los jóvenes en México. Región y Sociedad, 25(58): 165-202.

Rubio, Jesús. 2017. Sindicalización y precariedad laboral en México. Revista Región y Sociedad, 29(68): 37-75. http://dx.doi.org/10.22198/rys.2017.68.a247

Tomasina, Fernando. 2012. Los problemas en el mundo del trabajo y su impacto en salud.

Crisis financiera actual. Revista de Salud Pública, 14(1): 56-67. https://doi.org/10.1590/s012400642012000700006

Samaniego, Norma. 2010. El empleo y la crisis, precarización y nuevas "válvulas de escape". Economía UNAM, 7: 58-70.

Solís, Marlene. 2014. La precarización del trabajo desde una perspectiva sociocultural en un contexto fronterizo. Región y Sociedad, 26(59): 81-112. https://doi.org/10.22198/rys.2014.59.a74

Zúñiga, Marjorie. 2012. Flexibilización laboral y ética empresarial. Revista de derecho, 27: 321-349.

\section{Anexos}

Cuadro A1. Resultados estadísticos de la precariedad en México para 2005 y 2015

\begin{tabular}{|c|c|c|c|c|c|c|c|c|}
\hline & \multicolumn{2}{|c|}{ Nada precariedad } & \multicolumn{2}{|c|}{ Baja precariedad } & \multicolumn{2}{|c|}{ Media precariedad } & \multicolumn{2}{|c|}{ Alta precariedad } \\
\hline & 2005 & 2015 & 2005 & 2015 & 2005 & 2015 & 2005 & 2015 \\
\hline \multicolumn{9}{|c|}{ Sexo } \\
\hline Hombre & 3.4 & 2.2 & 48.9 & 40.2 & 14.1 & 19 & 33.6 & 38.6 \\
\hline Mujer & 5.2 & 2.8 & 49.9 & 43.8 & 11.9 & 18.2 & 33 & 35.2 \\
\hline \multicolumn{9}{|c|}{ Estado Civil } \\
\hline No unidos & 3.4 & 1.9 & 41.9 & 33.1 & 15.2 & 20.1 & 39.5 & 44.9 \\
\hline Unidos & 4.3 & 2.8 & 54.2 & 47.2 & 12.1 & 17.3 & 29.4 & 32.7 \\
\hline \multicolumn{9}{|c|}{ Edad } \\
\hline 15-19 & 1.7 & 1.4 & 40.4 & 29 & 14.5 & 19.1 & 43.4 & 50.5 \\
\hline $20-29$ & 2.6 & 1.8 & 45.2 & 34.7 & 14.2 & 19.3 & 38.1 & 44.3 \\
\hline $30-39$ & 4 & 2.3 & 49.6 & 40.6 & 13.4 & 18.9 & 33 & 38.2 \\
\hline $40-49$ & 6.1 & 3 & 53.2 & 46.7 & 12.4 & 17.9 & 28.3 & 32.4 \\
\hline $50-59$ & 9.2 & 3.9 & 55.5 & 52.5 & 11.3 & 16.4 & 24 & 27.2 \\
\hline 60 y más & 13.6 & 5 & 56.2 & 58 & 9.9 & 14.6 & 20.2 & 22.5 \\
\hline \multicolumn{9}{|c|}{ Escolaridad } \\
\hline Ninguno & 0 & 0 & 15.6 & 9.4 & 8.1 & 13.4 & 75.9 & 76.8 \\
\hline Básica & 1.8 & 1.2 & 36.1 & 25.4 & 13.5 & 19.6 & 48.7 & 53.9 \\
\hline Media superior & 7.3 & 3.6 & 58.8 & 50.9 & 11.6 & 16.4 & 22.2 & 29.2 \\
\hline Superior & 25.1 & 10.5 & 61.2 & 69.3 & 5.8 & 7.5 & 7.9 & 12.7 \\
\hline Posgrado & 58.6 & 27.1 & 36.6 & 65.8 & 2.2 & 2.2 & 2.5 & 4.9 \\
\hline
\end{tabular}


- Revista de Ciencias Sociales y Humanidades. ISSN-P: 0188-9834 ISSN-E: 2395-8669.

\begin{tabular}{|c|c|c|c|c|c|c|c|c|c|}
\hline \multicolumn{9}{|c|}{ Empleados } \\
\hline 1 persona & 0.8 & 0.3 & 4.8 & 3.5 & 8.4 & 8.6 & 86 & 87.7 \\
\hline $2-5$ personas & 1.4 & 0.6 & 12.8 & 9.8 & 13.8 & 15.4 & 72 & 74.1 \\
\hline $6-10$ personas & 2.4 & 1.3 & 29.2 & 24.6 & 16.6 & 20.4 & 51.9 & 53.7 \\
\hline $11-15$ personas & 4.1 & 3 & 52.1 & 48.3 & 12.7 & 16.9 & 31.1 & 31.8 \\
\hline $16-50$ personas & 7 & 6.4 & 71.1 & 69.5 & 6 & 8.2 & 15.9 & 15.9 \\
\hline 51 y más personas & 11.8 & 13.4 & 79.1 & 77.1 & 1.8 & 2.5 & 7.3 & 7.1 \\
\hline \multicolumn{3}{|c|}{} & & Sector & & & & \\
\hline Construcción & 5.5 & 4.1 & 58.8 & 52.2 & 11.8 & 20.2 & 23.9 & 23.5 \\
\hline Industria Manufacturera & 4.7 & 3.3 & 55 & 47.8 & 12.6 & 20.1 & 27.7 & 28.8 \\
\hline Comercio & 4.1 & 2.6 & 50.8 & 43.3 & 13.3 & 19.3 & 31.8 & 34.7 \\
\hline Servicios & 3.5 & 2.1 & 46.5 & 38.7 & 13.8 & 18 & 36.2 & 41.2 \\
\hline Otros & 3 & 1.7 & 42.1 & 34.2 & 14 & 16.1 & 40.8 & 48 \\
\hline Agropecuario & 2.6 & 1.3 & 37.8 & 29.9 & 14 & 13.9 & 46.7 & 54.9 \\
\hline
\end{tabular}

Fuente: Elaboración propia con base en datos de la ENOE

Cuadro A2. Probabilidades marginales de los niveles de precariedad laboral en México para 2005 y 2015

\begin{tabular}{|c|c|c|}
\hline \multicolumn{3}{|c|}{ Coeficientes } \\
\hline & 2005 & 2015 \\
\hline \multicolumn{3}{|c|}{ Nada de precariedad } \\
\hline Educación & $0.05^{*}$ & $0.03 *$ \\
\hline Sexo & $0.02 *$ & $0.01 *$ \\
\hline Estado civil & $0.01 *$ & $0.01 *$ \\
\hline Sector & $-0.01 *$ & $-0.01 *$ \\
\hline Empleados & $0.02 *$ & $0.02 *$ \\
\hline Edad & $0.02 *$ & $0.01^{*}$ \\
\hline \multicolumn{3}{|c|}{ Baja Precariedad } \\
\hline Educación & $0.24 *$ & $0.27 *$ \\
\hline Sexo & $0.01 *$ & $0.04 *$ \\
\hline Estado civil & $0.12 *$ & $0.14 *$ \\
\hline Sector & $-0.04 *$ & $-0.05 *$ \\
\hline Empleados & $0.23 *$ & $0.25 *$ \\
\hline Edad & $0.04 *$ & $0.06 *$ \\
\hline
\end{tabular}


- Revista de Ciencias Sociales y Humanidades. ISSN-P: 0188-9834 ISSN-E: 2395-8669.

\begin{tabular}{|c|c|c|}
\hline \multicolumn{3}{|c|}{ Media Precariedad } \\
\hline Educación & $-0.02^{*}$ & $-0.05^{*}$ \\
\hline Sexo & $-0.02^{*}$ & $-0.01^{*}$ \\
\hline Estado civil & $-0.03^{*}$ & $-0.03^{*}$ \\
\hline Sector & $0.01^{*}$ & $-0.01^{*}$ \\
\hline Empleados & $-0.06^{*}$ & $-0.06^{*}$ \\
\hline Edad & $-0.01^{*}$ & $-0.01^{*}$ \\
\hline & Alta Precariedad & \\
\hline Educación & $-0.27^{*}$ & $-0.24^{*}$ \\
\hline Sexo & $-0.01^{*}$ & $-0.03^{*}$ \\
\hline Estado civil & $-0.10^{*}$ & $-0.12^{*}$ \\
\hline Sector & $0.04^{*}$ & $0.06^{*}$ \\
\hline Empleados & $-0.19^{*}$ & $-0.21^{*}$ \\
\hline Edad & $-0.05^{*}$ & $-0.06^{*}$ \\
\hline
\end{tabular}

Fuente: Elaboración propia con base en datos de la ENOE 2005 y 2015.

* significativos al 95 por ciento de confianza.

\section{Gráfico I. Probabilidad de precariedad por sexo}

(a) 2005

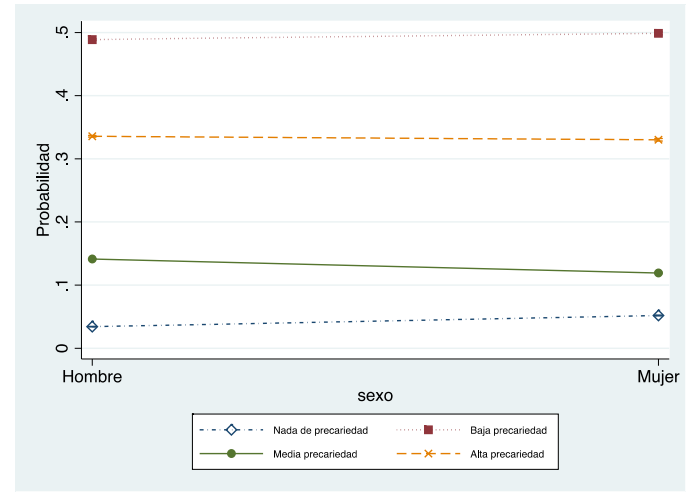

Fuente: Elaboración propia con base en datos de la ENOE. (b) 2015

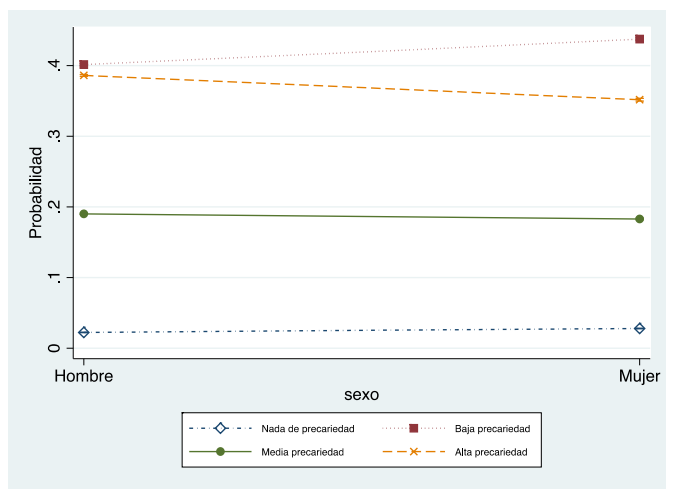

Fuente: Elaboración propia con base en datos de la ENOE. 


\section{Gráfico II. Probabilidad de precariedad por estado civil}

(a) 2005

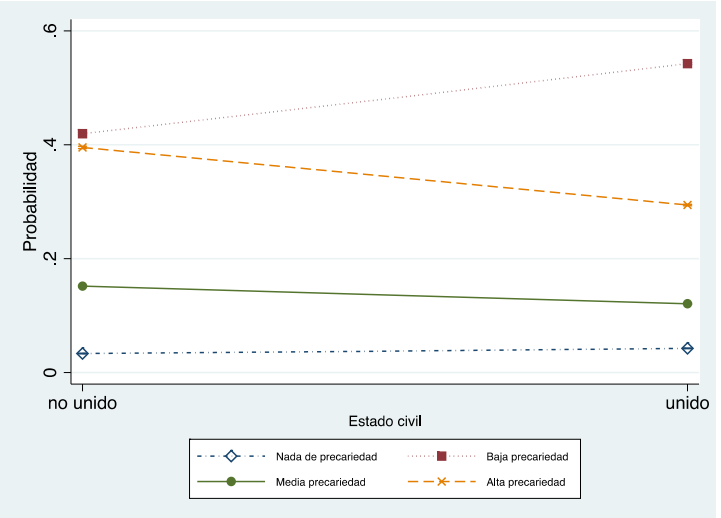

Fuente: Elaboración propia con base en datos de la ENOE. (b) 2015

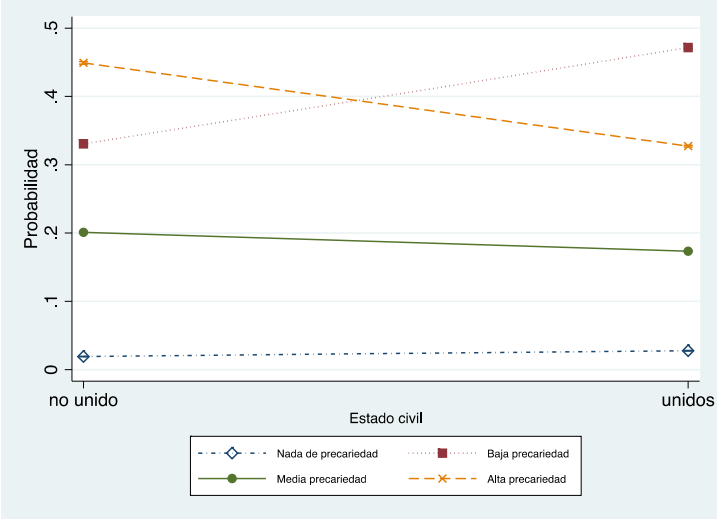

Fuente: Elaboración propia con base en datos de la ENOE.

\section{Gráfico III. Probabilidad de precariedad por rango de edad}

(a) 2005

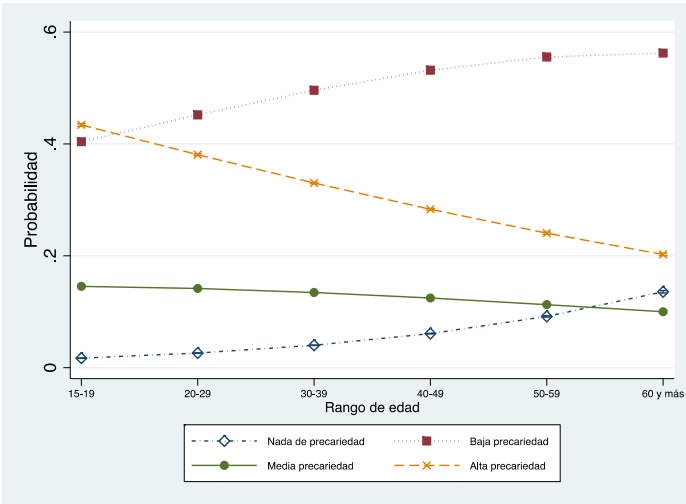

Fuente: Elaboración propia con base en datos de la ENOE. (b) 2015

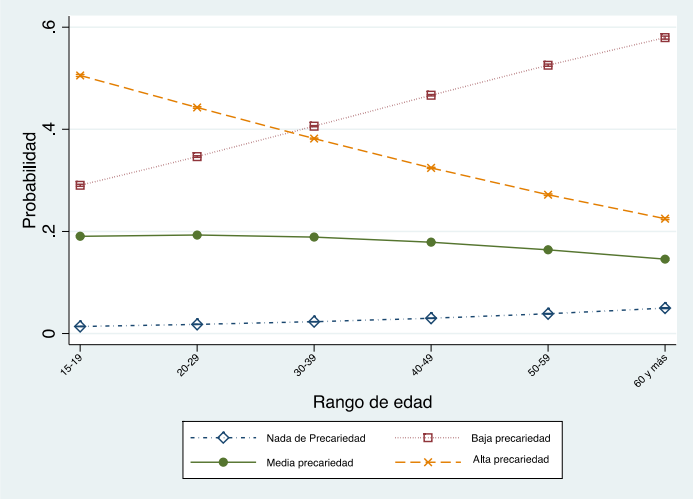

Fuente: Elaboración propia con base en datos de la ENOE. 


\section{Gráfico IV. Probabilidad de precariedad por escolaridad}

(a) 2005

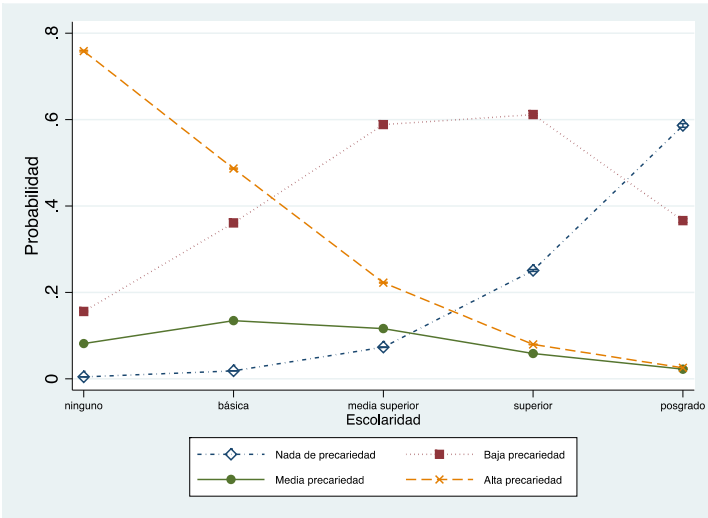

Fuente: Elaboración propia con base en datos de la ENOE. (b) 2015

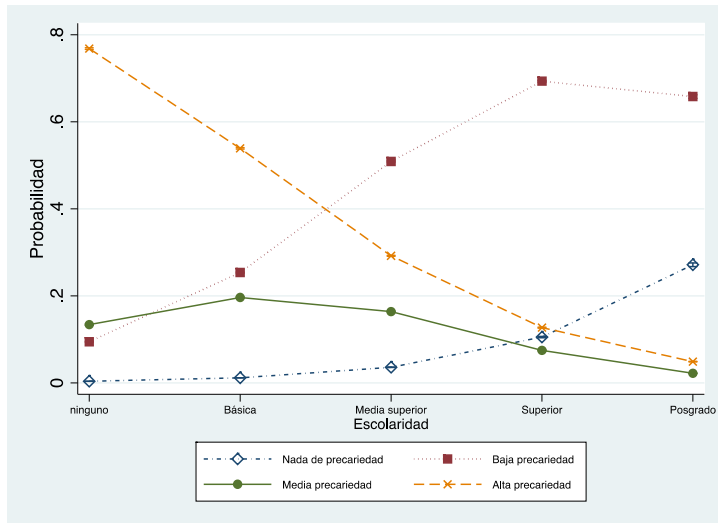

Fuente: Elaboración propia con base en datos de la ENOE.

Gráfico V. Probabilidad de precariedad por número de empleado

(a) 2005

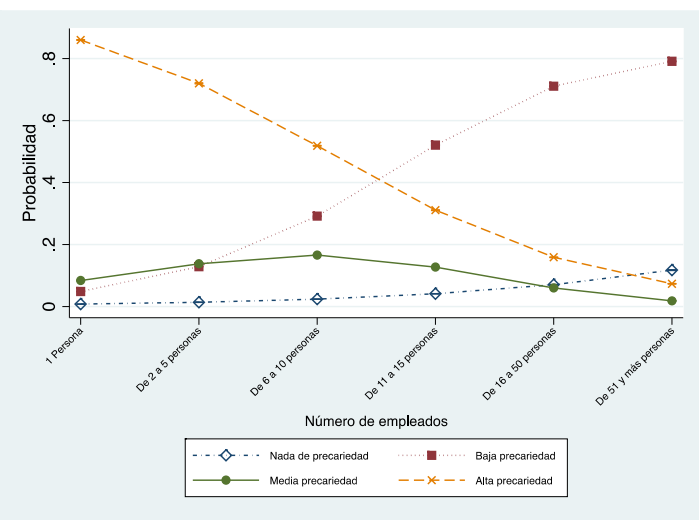

Fuente: Elaboración propia con base en datos de la ENOE. (b) 2015

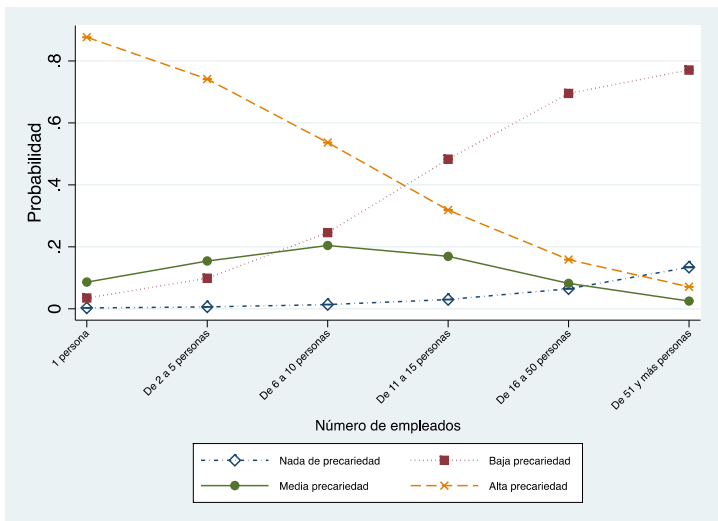

Fuente: Elaboración propia con base en datos de la ENOE. 


\section{Gráfico VI. Probabilidad de precariedad por sector}

(a) 2005

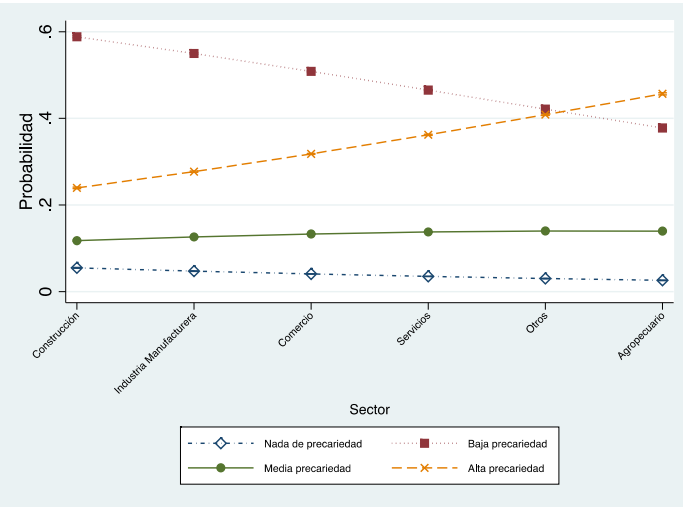

Fuente: Elaboración propia con base en datos de la ENOE. (b) 2015

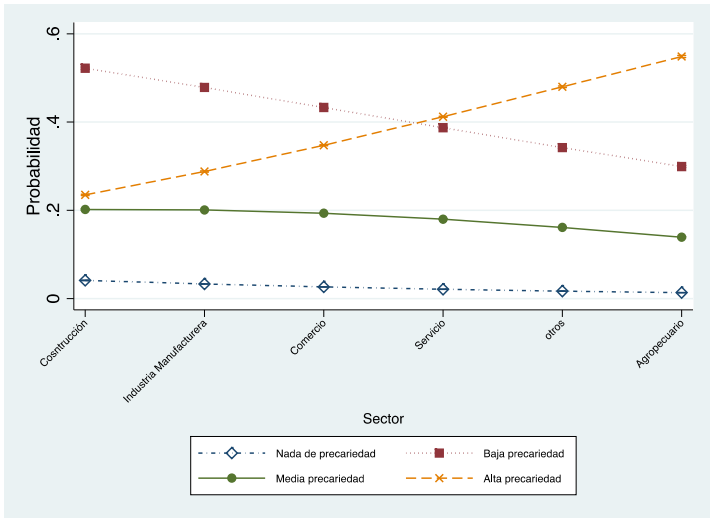

Fuente: Elaboración propia con base en datos de la ENOE. 